\title{
Factores asociados a la violencia conyugal en el Centro Emergencia Mujer-Lima
}

\section{Raúl Arce Cano ${ }^{1}$ Emma Norma Cambillo Moyano ${ }^{2}$}

Resumen: El objetivo de la investigación fue determinar los factores asociados con la violencia conyugal en el Centro de Emergencia Mujer (CEM) en los meses de enero hasta agosto en el año 2017. Para ello se realizó una investigación no experimental, descriptiva con diseño transversal, donde la población de estudio estuvo constituida por personas que sufrieron algún tipo de violencia y fueron atendidas en el CEM sede central, Lima en el año 2017 en los meses de enero hasta agosto, la muestra fue probabilística y comprendió 186 personas. Para el análisis bivariado se aplicó la prueba de Chi-cuadrado, del mismo modo se empleó la regresión logística binaria para calcular Odds Ratio (OR) con intervalos de confianza al 95\%. Los factores que resultaron significativos con la violencia conyugal son el factor psicológico y la ocupación de las víctimas, se muestra que las con trastorno de personalidad tienen 5 veces más chance de sufrir violencia sexual que las personas que sufren autoestima inadecuada, así como las personas con trastornos de personalidad tienen 16 veces más posibilidad de sufrir violencia psicológica que las personas que tienen autoestima baja.

Palabras clave: Violencia conyugal; Factor psicológico; factor sociológico.

\section{Factors associated with conjugal violence in the emergency center woman}

\begin{abstract}
The objective of the investigation was to determine the factors associated with conjugal violence are determined in the Women Emergency Center (CEM) in the months of January to August in the year 2017. For this a not experimental researches descriptive with transversal design was carried out, where the study population was constituted by people who suffered some type of violence and were treated at the central headquarters CEM, Lima in 2017 in the months of January to August, the sample was probabilistic and comprised 186 people. For the bivariate analysis, the Chi-square test was applied in the same way the binary logistic regression was used to calculate Odds Ratio (OR) with $95 \%$ confidence intervals. The factors that were affected with the conjugal violence are the psychological factor and the occupation of the victims, it is shown that those with personality disorder are 5 times more likely to suffer sexual violence than people who have inadequate self-esteem, as well as people with personality disorders are 16 times more likely to suffer psychological violence than people who have low self-esteem.
\end{abstract}

Keywords: Conjugal violence; psychological factor; sociological factor.

Recibido: 12/07/2019. Aceptado: 20/10/2019. Publicado online: 26/12/2019.

(CLos autores. Este artículo es publicado por la Revista PESQUIMAT de la Facultad de Ciencias Matemáticas, Universidad Nacional Mayor de San Marcos. Este es un artículo de acceso abierto, distribuido bajo los términos de la licencia Creative Commons Atribucion-No Comercia-Compartir Igual 4.0 Internacional.(http://creativecommons.org/licenses/by-nc-sa/4.0/) que permite el uso no comercial, distribución y reproducción en cualquier medio, siempre que la obra original sea debidamente citada. Para información, por favor póngase en contacto con revistapesquimat.matematica@unmsm.edu.pe

${ }^{1}$ UNMSM, Facultad de Ciencias Matemáticas e-mail: arceraul1@hotmail.com

${ }^{2}$ UNMSM, Facultad de Ciencias Matemáticas. e-mail: ecambillom@unmsm.edu.pe 


\section{Introducción}

La violencia conyugal o también denominada violencia de pareja o marital es una de las maneras más comunes o recurrentes de llevar a cabo acciones coercitivas entre miembros de una pareja, que puede ser heterosexual u homosexual, donde este fenómeno psicosocial puede incidir en ambos sexos (femenino o masculino). Sin embargo, de acuerdo a las estadísticas, estudios e investigaciones, existe una mayor incidencia de hombres agresores que de mujeres $[4,6,1]$. Al analizar la información obtenida por las denuncias de mujeres víctimas de violencia, la prevalencia de violencia de pareja no es considerado por los encuestados [8].

En el mundo se dio la "Cumbre del Milenio de las Naciones Unidas" (2000), donde se planteó como objetivo del desarrollo del milenio "promover el paralelismo para los géneros y la independencia de la mujer", y una de las principales metas era disminuir la cantidad de casos de "violencia conyugal", para lo cual era muy relevante conocer los factores que la causan. Asimismo, la violencia conyugal está considerada como un conflicto peligroso de salud pública que constituye un hecho social cuyas estadísticas tienen una tendencia al incremento en el Perú; no obstante, esta misma tendencia existe en el mundo.

En el Perú existe el Programa Nacional contra la Violencia Familiar y Sexual (PNCVFS), que es una Unidad Ejecutora del Ministerio de la Mujer y Poblaciones Vulnerables (MIMP), de acuerdo con la Ley No 29247, creado a través del Decreto Supremo N ${ }^{\circ}$ 008-2001-PROMUDEH, teniendo su sede central en la ciudad de Lima desarrollando sus actividades en todo el país, como un organismo público rector de las Políticas Nacionales y Sectoriales para la Mujer y Localidades Sensibles. Asimismo, la finalidad del PNCVFS es ayudar a la disminución de la elevada prevalencia de la intimidación familiar en mujeres niñas, niños y adolescentes; manifestando, esbozando, originando y ejecutando técnicas, proyectos y programas de previsión y atención, igualmente de originar ejercicios de amparo y recuperación para la violencia familiar y sexual; así como la reproducción del discernimiento de la dificultad y la promoción de las vinculaciones democráticas entre varones y mujeres. Además, la PNCVFS interviene por intermedio de tres Líneas de acción: $1^{o}$ Prevención para la Violencia familiar y sexual y promoción de relaciones democráticas entre varones y mujeres; $2^{\circ}$ Cuidado dominado para la violencia familiar y sexual; y $3^{\circ}$ Reproducción de indagación y gestión del discernimiento.

Los Centros Emergencia Mujer son unidades orgánicas dependientes del Programa Nacional Contra la Violencia Familiar y Sexual que brindan servicios públicos especializados para prestar atención a las víctimas de violencia familiar y sexual cumplen un papel catalizador para lograr la adecuada y oportuna atención, en las instancias pertinentes, de las personas implicadas en actos de violencia familiar y sexual, tanto para canalizarla a las instancias legales, como para la rehabilitación psicosocial. Actualmente, hay 200 CEM aproximadamente en el país, sin embargo, ellos no cubren a todo el territorio nacional a nivel provincial, y su cobertura a nivel distrital es limitada, porque su cobertura nacional es del $0,7 \%$ [9].

El presente trabajo de investigación aborda la identificación de factores que están asociados a la violencia conyugal y a cada tipo de violencia por separado en las personas que acudieron al CEM en los meses de enero a agosto en el 2017, para este análisis usamos el modelo de regresión logística. Por lo tanto, el presente trabajo se abordará los "Factores asociados a la violencia conyugal en el centro emergencia mujer - CEM, Lima, 2017".

\section{Metodología}

El tipo de investigación es no experimental o ex post facto, ya que el investigador no manipula la variable independiente (factores), ni la variable dependiente (violencia conyugal); dado que 
los hechos de la conducta agresiva y abusiva ocurre en un contexto relación heterosexual, donde no se puede controlar el comportamiento que habitualmente se sucede en una relación de pareja donde por acción u omisión se perjudica física, psicológica o sexualmente al otro miembro.

El diseño de la investigación es descriptivo, porque detalla las características, costumbres, datos, objetos, personas y/o procesos de la violencia conyugal y como esta afecta a la vida de las mujeres, que son las que sufren mayormente las conductas agresivas, y con esta información se podrá saber el por qué y para qué se está investigando.

El diseño de investigación es causal, porque permitirá apreciar el grado de relación existente entre las dos variables de investigación: Variable Independiente = Principales Factores, y Variable Dependiente $=$ Violencia Conyugal.

\section{Unidad de análisis}

Persona que ha sido atendida en el CEM con algún tipo de violencia (tanto para hombres o mujeres), cuya característica fundamental es una estrecha relación de dependencia o subordinación entre agredida y agresor, donde la víctima permanece al lado del victimario a pesar de ser violentada durante largos períodos de tiempo; y existe una indefinición y una vulneración de los derechos de la víctima y, también, un control intencionado de dominación del hombre.

La población son todas las personas atendidas en el Centro Emergencia Mujer-CEM, Sede Central, Lima en el año 2017, determinado según cuadro:

Cuadro 1. Casos atendidos por algún tipo de violencia en el CEM en los meses de enero hasta agosto en el año 2017

\begin{tabular}{|c|c|}
\hline Meses & Cantidad \\
\hline Enero & 174 \\
Febrero & 169 \\
Marzo & 203 \\
Abril & 178 \\
Mayo & 147 \\
Junio & 235 \\
Julio & 198 \\
Agosto & 230 \\
\hline Total & 1534 \\
\hline
\end{tabular}

El marco muestral lo constituyen todos los registros de violencia conyugal atendidos en el CEM de Lima en el año 2017 durante los meses de enero a agosto.

Para obtener el tamaño de muestra, consideramos el tipo probabilístico de selección sistemática de las fichas de evaluación de las víctimas de violencia conyugal registrados en CEM, Lima 2017.

Para lo cual el tamaño de muestra se obtendrá empleando el siguiente criterio:

a) El nivel de confiabilidad de la muestra: $95 \%$

b) El margen de error estimado: $\mathrm{e}=5 \%$

c) Proporción de personas que han sufrido violencia física frente a otros que han sufrido violencia conyugal: $p=70 \%$

d) La población es finita: $N=1534$ 
Para la selección de la muestra utilizaremos el muestreo sistemático [7] y se seguirá los siguientes pasos:

a) Se elabora una lista ordenada de $N=1534$ fichas.

b) Calculamos el tamaño del salto sistemático:

$$
K=\frac{N}{n}=\frac{1534}{186}=8,25 \approx 8 \Rightarrow K=8
$$

c) Número de inicio: Seleccionamos un número aleatorio entero entre 1 y 8. Sea el numero aleatorio: $h=6$ (Este número corresponderá a la $1^{\text {ra }}$ persona que es víctima de Violencia conyugal, que es seleccionado en la muestra).

d) Selección de los individuos restantes.

$$
6,6+1 \times 8,6+2 \times 8,6+3 \times 8,6+4 \times 8, \ldots, 6+135 \times 8
$$

Es decir: $6,14,22,30,38, \ldots, 1086$ que constituye los $n=186$ elementos de la muestra.

Las variables que se utilizó en la investigación, son las siguientes:

\section{Variable dependiente}

- Violencia conyugal. Esta variable se divide en dos categorías: las personas que han sufrido violencia conyugal (física, sexual o psicológica) y aquellas que han sufrido algún otro tipo de violencia. Para fines de la investigación se ha codificado como $1=$ Sufrió violencia conyugal, $0=$ sufrió otro tipo de violencia.

\section{Variable independientes}

Las variables independientes utilizadas en la investigación de acuerdo al marco conceptual son:

Sexo. Es una variable que tiene las siguientes categorías: femenino y masculino. Su codificación es la siguiente: $1=$ mujer y $2=$ varón.

Edad. Es una variable transformada que tiene como variable origen "la edad en años de la víctima de violencia". Cabe indicar que esta variable inicialmente es cuantitativa, luego fue transformada para fines del procesamiento y análisis e interés de la propia investigación. Su codificación es la siguiente: $1=$ menor o igual a 35 años y $2=$ mayor a 35 años.

\section{Factor educativo.}

- Grado de instrucción. Es una variable transformada que tiene como variable origen "el Nivel de instrucción alcanzado". Es el año o grado de estudios más alto aprobado por cada persona dentro del nivel de educación alcanzado. Comprende las siguientes categorías: sin nivel (analfabetos), primaria, secundaria, técnico y superior. Cabe indicar que esta variable fue transformada para fines del procesamiento y análisis e interés de la propia investigación en tres categorías; $0=$ analfabetos o primaria completa; $1=$ secundaria y $2=$ técnico superior completo.

\section{Factor económico.}

- Ocupación. Es una variable transformada que tiene como variable origen "la ocupación actual de la víctima de violencia". Su codificación es la siguiente: $0=$ otras ocupaciones; $1=$ trabajadora dependiente y $2=$ trabajadora independiente sector privada. 


\section{Factor psicológico}

- Trastornos psicológicos. Es una variable transformada que tiene como variable origen los trastornos psicológicos. Comprende las siguientes categorías: autoestima inadecuada, celotipia, trastornos de personalidad y alcoholismo y drogadicción. Cabe indicar que esta variable fue transformada para fines del procesamiento y análisis e interés de la propia investigación. Su codificación es la siguiente: $0=$ autoestima inadecuada; $1=$ celotipia y trastornos de personalidad; 2 = alcoholismo y drogadicción.

\section{Factor sociológico}

- Conductas sociológicas. Es una variable transformada que tiene como variable origen las conductas sociológicas de la persona. Comprende las siguientes categorías: familia patriarcal, machismo, influencia de medios masivos e incomunicación conyugal. Cabe indicar que esta variable fue transformada para fines del procesamiento y análisis e interés de la propia investigación. Su codificación es la siguiente: $0=$ familia patriarcal y machismo; $1=$ influencia de medios masivos y $2=$ incomunicación conyugal.

Cuadro 2. Variables del factor sociológico.

\begin{tabular}{|l|l|l|}
\hline Variables & Categorías & Tipo \\
\hline Sexo & $\begin{array}{l}\text { Femenino } \\
\text { Masculino }\end{array}$ & $\begin{array}{l}\text { Cualitativa } \\
\text { Nominal }\end{array}$ \\
\hline Edad & $\begin{array}{l}\text { Menor o igual a 35 años } \\
\text { Mayor a 35 años }\end{array}$ & $\begin{array}{l}\text { Cualitativa } \\
\text { Ordinal }\end{array}$ \\
\hline $\begin{array}{l}\text { Grado de } \\
\text { instrucción } \\
\text { (concluido) }\end{array}$ & $\begin{array}{l}\text { Analfabeta-primaria } \\
\text { Secundaria } \\
\text { Técnico-Superior }\end{array}$ & $\begin{array}{l}\text { Cualitativa } \\
\text { Ordinal }\end{array}$ \\
\hline Ocupación & $\begin{array}{l}\text { Dependiente } \\
\text { Independiente } \\
\text { Otras }\end{array}$ & $\begin{array}{l}\text { Cualitativa } \\
\text { Nominal }\end{array}$ \\
\hline $\begin{array}{l}\text { Trastornos } \\
\text { psicológicos }\end{array}$ & $\begin{array}{l}\text { Autoestima inadecuada } \\
\text { Trastornos de personalidad } \\
\text { Alcoholismo y drogadicción }\end{array}$ & $\begin{array}{l}\text { Cualitativa } \\
\text { Nominal }\end{array}$ \\
\hline $\begin{array}{l}\text { Conductas } \\
\text { sociológicas }\end{array}$ & $\begin{array}{l}\text { Familia patriarcal } \\
\text { Influencia de medios masivos } \\
\text { Incomunicación conyugal }\end{array}$ & $\begin{array}{l}\text { Cualitatica } \\
\text { Nominal }\end{array}$ \\
\hline $\begin{array}{l}\text { Tipos de } \\
\text { discriminación } \\
\text { de género }\end{array}$ & $\begin{array}{l}\text { Mujer como objeto sexual } \\
\text { Mujer como propiedad priva- } \\
\text { da } \\
\text { Discriminación de la mujer } \\
\text { por género }\end{array}$ & $\begin{array}{l}\text { Cualitativa } \\
\text { Nominal }\end{array}$ \\
\hline
\end{tabular}

\section{Factor cultural}

- Discriminación de género. Comprende las siguientes categorías: $0=$ discriminación de la mujer por género; $1=$ mujer como objeto sexual y $2=$ mujer como propiedad privada.

\section{Procedimientos y técnicas de recolección de Datos}

Los datos fueron recolectados por el investigador en los ambientes del CEM, en un periodo de tiempo de 15 días, a partir del registro se elaboró la base de datos en SPSS. 


\section{Ficha de Admisión del Centro de Emergencia Mujer - CEM}

La cédula del cuestionario constituye uno de los instrumentos para la recopilación de datos de la presente investigación. Asimismo, este cuestionario es un listado de preguntas ordenadas sistemáticamente de acuerdo a los objetivos del estudio de las variables de investigación.

\section{Plan de análisis}

Con la finalidad de alcanzar los objetivos propuestos en la investigación fue necesario elaborar una base de datos. Se relata brevemente los procedimientos realizados para obtener la base de datos:

Primero se recolectaron los datos de las fichas en los ambientes del CEM.

Segundo a partir de la base ya creada con información se diseñó y organizó una sola base de datos a nivel nominal, conteniendo información sobre variables relacionadas a la violencia conyugal organizadas de acuerdo al objetivo de la investigación.

Tercero para describir el perfil de la población que sufre de violencia conyugal, que acude al CEM centro Lima durante los meses de enero hasta agosto 2017, se realizó un análisis estadístico descriptivo e inferencial, con la finalidad de describir las características que influyen como factores de violencia como: la edad, años de estudios alcanzados, ocupación, trastornos psicológicos , conductas sociológicas y tipos de discriminación.

Cuarto para identificar y establecer la relación entre factores educativo, psicológico, económico, sociológico y cultural asociados a la violencia conyugal durante los meses de enero hasta agosto el año 2017 se utilizaron los modelos de respuesta binaria. Es decir, se realizó un análisis bivariado entre la variable dependiente violencia conyugal y cada una de las variables independientes que representan con la finalidad de identificar, en un primer nivel de análisis, la asociación entre la variable dependiente y las variables independientes así como definir las variables que deberán ingresar en el análisis multivariado.

Los modelos de respuesta binaria se utilizan de manera frecuente cuando la variable dependiente es dicotómica; por ejemplo, si las personas sufrieron de violencia conyugal o no en el año 2017 en el CEM centro Lima.

Una ventaja de los modelos de respuesta binaria es que se pueden interpretar en función de los odds ratios (razón de chance), principal medida de relación entre dos variables dicotómicas.

Para la preparación de la base de datos y para el análisis de los modelos de respuesta binaria se utilizaron los programas estadísticos IBM SPSS versión 24 (en español).

\section{Resultados}

El estudio se realizó con una muestra sistemática de 186 personas que sufrieron algún tipo de violencia y fueron atendidos en el Centro emergencia Mujer (CEM) centro Lima en el año 2017 en los meses de enero hasta agosto.

\subsection{Análisis descriptivo univariado}

La mayoría de las personas atendidas $(65 \%)$ han sufrido tres tipos de violencia: física, psicológica y sexual, un $28 \%$ de las 186 han sufrido dos tipos de violencia, el $3 \%$ ha sufrido solo violencia psicológica, otro $3 \%$ ha sufrido solo violencia sexual y un $1 \%$ solo violencia física. 
Cuadro 3. Distribución de los tipos de violencia dados en el CEM Lima Centro. Enero - Agosto 2017.

\begin{tabular}{|l|c|c|}
\hline Tipo de violencia & Frecuencia & Porcentaje \\
\hline Física + Psicológica + Sexual & 120 & $65 \%$ \\
Física + Psicológica & 19 & $10 \%$ \\
Psicológica + Sexual & 17 & $9 \%$ \\
Física + Sexual & 16 & $9 \%$ \\
Violencia psicológica & 6 & $3 \%$ \\
Violencia sexual & 6 & $3 \%$ \\
Violencia física & 2 & $1 \%$ \\
\hline Total & $\mathbf{1 8 6}$ & $\mathbf{1 0 0 \%}$ \\
\hline
\end{tabular}

Con respecto a las características demográficas de las personas atendidas, se observa que la mayoría de las victimas $(55 \%)$ tienen menos o igual a 35 años. En cuanto al género la mayoría de víctimas (98\%), de algún tipo de violencia son mujeres. (Cuadro 4).

Cuadro 4. Características demográficas de las víctimas de algún tipo de violencia dados en el CEM Lima Centro. Enero - Agosto 2017. $(n=186)$.

\begin{tabular}{|l|c|c|}
\hline Característica & Cantidad & Porcentaje \\
\hline Edad & 102 & $55 \%$ \\
Menor o igual a 35 años & 84 & $45 \%$ \\
Mayor a 35 años & 182 & \\
Sexo & 4 & $98 \%$ \\
Femenino & & $2 \%$ \\
Masculino & & \\
\hline
\end{tabular}

En cuanto a la frecuencia de ocurrencia de los diferentes factores, se aprecia que en el factor educativo la mayoría de víctimas son personas que han acabado los estudios superiores o técnicos $(38 \%)$. En cuanto al factor psicológico se observa que la mayoría de personas que han sufrido algún tipo de violencia son aquellas que presentan algún trastorno de personalidad (51\%); en lo que respecta al factor sociológico vemos que la mayoría de víctimas de algún tipo de violencia son personas con una familia patriarcal o machismo (52\%); en el factor cultural la mayoría de víctimas son mujeres como objeto sexual 83 casos (45\%). Y por último en cuanto a ocupación de la víctima vemos que la mayoría son trabajadores dependientes estatales (Cuadro 5).

\subsection{Análisis bivariado: Factores asociados a la violencia conyugal, física, psi- cológica y sexual}

En esta etapa se analizó la existencia de posibles relaciones entre los factores y variables demográficas con la violencia conyugal, física, psicológica y sexual. Este análisis puede realizarse desde una óptica bivariada, en nuestro caso cuando las variables son cualitativas, se utilizó una tabla de contingencia, a partir de dicha tabla podemos estudiar si las dos variables están o no asociadas y evaluar su grado de asociación siempre y cuando ambas variables tengan dos categorías cada una. 
Cuadro 5. Víctimas de algún tipo de violencia según factores dados en el CEM Lima Centro. Enero - Agosto 2017. $(n=186)$

\begin{tabular}{|l|c|c|}
\hline Factor & Cantidad & Porcentaje \\
\hline Factor educativo & 61 & $33 \%$ \\
Analfabeto-Primaria completa & 51 & $29 \%$ \\
Secundaria & 71 & $38 \%$ \\
Técnico superior & & \\
Factor psicológico & 31 & $17 \%$ \\
Autoestima inadecuada & 95 & $51 \%$ \\
Trastornos de personalidad & 60 & $32 \%$ \\
Alcoholismo y drogadicción & 97 & $52 \%$ \\
Factor sociológico & 37 & $20 \%$ \\
Familia patriarcal-Machismo & 52 & $28 \%$ \\
Influencia de medios masivos & & \\
Incomunicación conyugal & 83 & $45 \%$ \\
Factor cultural & 57 & $30 \%$ \\
Mujer como objeto sexual & 46 & $25 \%$ \\
Mujer como propiedad privada & & \\
Discriminación de la mujer por & & \\
género & 83 & $45 \%$ \\
Ocupación & 65 & $35 \%$ \\
Trabajador dependiente estatal & 38 & $20 \%$ \\
Trabajador independiente privada & & \\
Otras &
\end{tabular}

\subsection{Factores asociados a la violencia conyugal}

Los porcentajes de violencia conyugal según edad es muy parecido en ambos grupos, vemos que el porcentaje de víctimas de violencia conyugal en personas menores de 35 años es $65 \%$ y en mayores a 35 años es $64 \%$. En cuanto al género, todas las víctimas de violencia conyugal son mujeres, no hay ningún varón, víctima de violencia conyugal. La violencia conyugal se relaciona significativamente con el sexo de la víctima $p<0,05$. (Cuadro 6).

El mayor porcentaje de violencia conyugal se da en las mujeres con grado de instrucción analfabeta o primaria completa y también en mujeres con grado de instrucción técnico o superior $(70 \%)$. La mayor proporción de violencia conyugal se da en víctimas con otros tipos de ocupaciones $(74 \%)$. En cuanto al factor psicológico el mayor porcentaje de violencia conyugal se da en las mujeres con entornos de alcoholismo y drogadicción (87\%), respecto al factor sociológico el mayor porcentaje de violencia conyugal se da en las mujeres con incomunicación conyugal $(73 \%)$ y en el factor cultural la mayor proporción se da en las mujeres como objeto sexual $(72 \%)$.

La violencia conyugal se relaciona significativamente con el factor psicológico $p<0,05$. 
Cuadro 6. Datos demográficos y violencia conyugal. CEM Lima Centro.

Enero - Agosto 2017. $(n=186)$

\begin{tabular}{|l|c|c|c|c|c|}
\hline \multirow{2}{*}{ Datos demográficos } & \multicolumn{4}{|c|}{ Violencia Conyugal } & \multirow{2}{*}{$p^{(1)}$} \\
\cline { 2 - 5 } & \multicolumn{2}{|c|}{ No } & \multicolumn{2}{|c|}{ Si } & \\
\cline { 2 - 5 } & $n$ & $\%$ & $n$ & $\%$ & \\
\hline Edad & & & & & \multirow{2}{*}{ Menor de 35 años } \\
Mayor a 35 años & 36 & $35 \%$ & 66 & $65 \%$ & 0.952 \\
Sexo & & $36 \%$ & 54 & $64 \%$ & \\
Femenino & 62 & $34 \%$ & 120 & $66 \%$ & \multirow{2}{*}{0.006} \\
Masculino & 4 & $100 \%$ & 0 & $0 \%$ & \\
Total & 66 & $35 \%$ & 120 & $65 \%$ & \\
\hline
\end{tabular}

(1) Prueba Chi cuadrado, significativo $p<0,05$

\subsection{Modelo de regresión logística}

Se construyó un modelo de regresión logística, que permita explicar la violencia conyugal a partir de un conjunto de factores. Se propusieron cuatro modelos, tomando como variable respuesta a la violencia conyugal (víctimas que sufren los tres tipos de violencia), y luego un modelo para cada tipo de violencia: física, psicológica y sexual.

Los modelos fueron ajustados considerando todas las variables menos la variable sexo ya que al ajustar los modelo con esta variable, los valores de los coeficientes resultantes de las otras variables varían significativamente dando como resultado estimaciones imprecisas.

\section{Ajuste del modelo: Violencia Conyugal}

Luego de ajustar el modelo de regresión logística con todas las variables indicadas, se observa que el factor psicológico (autoestima inadecuada/ Trastornos de personalidad/Alcoholismo y drogadicción) y la ocupación de la víctima (Independiente) son los que explican significativamente $(p<0,05)$ la violencia conyugal (Cuadro 7).

$\mathrm{El}$ análisis de los coeficientes del modelo ajustado, permite deducir para el factor psicológico que las personas con trastornos de personalidad tienen mayor riesgo (Beta positivo $=1,23$ ) de sufrir violencia conyugal que las personas con autoestima inadecuada (categoría de referencia). Lo mismo sucede para las personas con entornos de alcoholismo o drogadicción, ellas tienen mayor chance (Beta positivo $=3,21$ ) de sufrir violencia conyugal que las personas con autoestima inadecuada.

En cuanto a la ocupación de la víctima decimos que aquellas que son trabajadores independientes tienen menos chance (Beta $=-1,15)$ de sufrir violencia conyugal que las personas que tienen otro tipo de ocupaciones (IC: 0,10-0,99). Es importante tener en cuenta que la categoría de la ocupación de la víctima dependiente no resultó significativa estadísticamente $(p=0,86)$. En el caso de las variables categóricas, si alguna categoría resulta significativa la variable queda incluida en el modelo final, aun cuando el resto no lo sea.

El odds de sufrir violencia conyugal comparado con el odds de no sufrir violencia conyugal, cuando la víctima tiene trastornos de personalidad es 3,4 veces mayor (IC: 1,29-9,05) comparado con una víctima con autoestima inadecuada. Similarmente el odds de sufrir violencia conyugal comparada con no sufrir violencia conyugal para alguna víctima en cuyo entorno hay alcohol y drogas es 24 veces mayor comparado con el de una víctima que presenta autoestima inadecuada (IC: $7,10-85,85)$. 
Cuadro 7. Factores asociados con la violencia conyugal en el modelo logístico. CEM Lima Centro. Enero - Agosto 2017. $(n=186)$

\begin{tabular}{|c|c|c|c|c|c|}
\hline Factores & Beta & $\mathrm{P}$ & OR & $\begin{array}{c}\text { I.C. } \\
95 \% \\
\text { Inferior }\end{array}$ & $\begin{array}{c}\text { Para } \\
\text { OR } \\
\text { Superior }\end{array}$ \\
\hline $\begin{array}{l}\text { Edad } \\
\text { Grado de instrucción(Analfabeto-primaria) }{ }^{1}\end{array}$ & $-0,15$ & 0,718 & 0,858 & 0,373 & 1,973 \\
\hline Secundaria & $-0,42$ & 0,422 & 0,656 & 0,234 & 1,837 \\
\hline Técnico-Superior & 0,32 & 0,495 & 1,384 & 0,544 & 3,521 \\
\hline $\begin{array}{l}\text { Factor psicológico(Autoestima inadecuada) }{ }^{1} \\
\text { psicológicos(trastornos de personalidad) }\end{array}$ & 1.23 & 0.014 & 3.415 & 1.288 & 9.051 \\
\hline psicológicos(Alcoholismo y drogadicción) & 3,21 & 0,000 & 24,695 & 7,103 & 85,858 \\
\hline $\begin{array}{l}\text { Factor sociológico(incomunicación conyugal })^{1} \\
\text { sociológicas(familia patriarcal) }\end{array}$ & -0.17 & 0.726 & 0.843 & 0.322 & 2.140 \\
\hline $\begin{array}{l}\text { sociológicas (influencia de medios masivos) } \\
\text { Factor cultural (mujer como objeto sexual) }{ }^{1}\end{array}$ & $-1,01$ & 0,109 & 0,368 & 0,108 & 1,248 \\
\hline Discriminación(Mujer como propiedad privada) & 0,14 & 0,784 & 1,1560 & 0,410 & 3,264 \\
\hline $\begin{array}{l}\text { Discriminación(por género) } \\
\text { Ocupación(otros })^{1}\end{array}$ & $-0,95$ & 0,063 & 0,386 & 0,149 & 1,078 \\
\hline Ocupación(Dependiente) & $-0,09$ & 0,869 & 0,914 & 0,314 & 2,662 \\
\hline Ocupación(Independiente) & $-1,15$ & 0,049 & 0,318 & 0,101 & 0,999 \\
\hline Coeficiente de determinación & \multicolumn{5}{|c|}{$32 \%$} \\
\hline Bondad de ajuste & \multicolumn{5}{|c|}{$\left(X^{2}=48,48 ; p=0,000\right)$} \\
\hline
\end{tabular}

(1) Categoría de referencia, significativo $p<0,05$

En cuanto a la ocupación de la víctima decimos que aquellas que son trabajadores independientes tienen menos chance de sufrir violencia conyugal que las víctimas que tienen otros tipos de ocupaciones (IC: 0,10-0,99).

El coeficiente de determinación indica que $32 \%$ de la variación en la violencia conyugal esta explicada por los factores incluidos en el modelo.

La prueba de bondad de ajuste del modelo $\left(X^{2}=48,48 ; p=0,000\right)$, indica que por lo menos uno de los factores antes mencionados explica significativamente la violencia conyugal.

La evaluación de la capacidad de pronóstico del modelo ajustado, indica que el $89 \%$ de las víctimas de violencia conyugal fueron clasificados correctamente como víctimas de violencia conyugal, mientras que el $53 \%$ de las personas que no han sufrido violencia conyugal fueron clasificados correctamente por el modelo. En resumen el modelo clasificó correctamente el $76 \%$ de las víctimas

\section{Ajuste del modelo: Violencia conyugal Física}

Luego de ajustar el modelo de regresión logística se observa que el grado de instrucción (analfabeta primaria completa/ Secundaria completa/ Técnico superior), el factor psicológico (autoestima inadecuada/ Trastornos de personalidad/Alcoholismo y drogadicción) y el factor sociológico (Familia patriarcal) son los que explican significativamente $(p<0,05)$ la violencia física.

El análisis de los coeficientes del modelo ajustado, permite deducir para el grado de instrucción que en las víctimas que tienen secundaria completa disminuye (Beta $=-1,99)$ el riesgo de sufrir violencia física en relación con las que son analfabetas o tienen primaria completa. 
Manteniendo constante el grado de instrucción y el factor sociológico, el odds de sufrir violencia física comparada con no sufrir violencia física para alguna víctima en cuyo entorno hay alcohol y drogas es 6 veces mayor comparado con el de una víctima que presenta autoestima inadecuada (IC: 1,18-31,03). La victima que proviene de familias patriarcales son aquellas que tienen más chance de sufrir violencia física que las victimas con incomunicación conyugal (IC: 4,06-157,14).

El coeficiente de determinación indica que $52 \%$ de la variación en la violencia física está explicada por los factores incluidos en el modelo.

La prueba de bondad de ajuste del modelo $\left(X^{2}=69,21 ; p=0,000\right)$, indica que por lo menos uno de los factores antes mencionados explica significativamente la violencia física.

La evaluación de la capacidad de pronóstico del modelo ajustado, indica que el $97 \%$ de las víctimas de violencia física fueron clasificados correctamente como víctimas de violencia física, mientras que el $61 \%$ de las personas que no han sufrido violencia física fueron clasificados correctamente por el modelo. En resumen el modelo clasificó correctamente el $91 \%$ de las víctimas de violencia física.

\subsubsection{Ajuste del modelo: Violencia conyugal Psicológica}

El modelo fue ajustado considerando todas las variables menos la variable sexo y el factor sociológico ya que al ajustar el modelo con estas variables, los valores de los coeficientes resultantes de las otras variables varían significativamente dando como resultado estimaciones imprecisas.

Luego de ajustar el modelo, el factor psicológico (autoestima inadecuada/ Trastornos de personalidad/Alcoholismo y drogadicción) y el factor cultural (Discriminación de la mujer por género) son los que explican significativamente ( $\left.\mathrm{p}_{\mathfrak{1} 0.05}\right)$ la violencia psicológica en las victimas.

El análisis de los coeficientes de las variables significativas del modelo ajustado, permite deducir para el factor psicológico que las víctimas con trastornos de personalidad tienen mayor riesgo (Beta positivo $=2,80$ ) de sufrir violencia psicológica que las personas con autoestima inadecuada (categoría de referencia). Lo mismo sucede para las personas con entornos de alcoholismo o drogadicción tienen mayor riesgo (Beta positivo=3,23) de sufrir violencia psicológica que las personas con autoestima inadecuada.

La víctima que es discriminada por su género tiene menos chance de sufrir violencia psicológica que las víctimas que son vistas como objeto sexual (IC: 0,04-0,76).

El coeficiente de determinación indica que el $49 \%$ de la variación en la violencia psicológica esta explicada por los factores incluidos en el modelo.

La prueba de bondad de ajuste del modelo $\left(X^{2}=56,57 ; p=0,000\right)$, indica que por lo menos uno de los factores antes mencionados explica significativamente la violencia psicológica.

La evaluación de la capacidad de pronóstico del modelo ajustado, indica que el $98 \%$ de las víctimas de violencia psicológica fueron clasificados correctamente como víctimas de violencia psicológica, mientras que el $50 \%$ de las personas que no han sufrido violencia psicológica fueron clasificados correctamente por el modelo. En resumen el modelo clasificó correctamente el $92 \%$ de las víctimas de violencia psicológica.

\section{Ajuste del modelo: Violencia conyugal Sexual}

Luego de ajustar el modelo, el factor psicológico, el factor sociológico (Influencia de medios masivos) y la ocupación de la víctima (Independiente) son los que explican significativamente $(p<0,05)$ la violencia sexual en las víctimas. 
$\mathrm{Al}$ analizar los resultados podemos deducir que manteniendo constante el factor sociológico y la ocupación de la víctima, el odds de sufrir violencia sexual comparado con el odds de no sufrir violencia sexual, cuando la víctima tiene trastornos de personalidad es 5 veces mayor (IC: 1,37 - 20,98) comparado con una víctima con autoestima inadecuada. Similarmente el odds de sufrir violencia sexual comparada con no sufrir violencia sexual para alguna víctima en cuyo entorno hay alcohol y drogas es 10 veces mayor comparado con el de una víctima que presenta autoestima inadecuada (IC: 6,83-35,99). La víctima que tiene influencia de medios masivos son aquellas que tienen menos chance de sufrir violencia sexual que las víctimas con incomunicación conyugal (IC: 0,01-0,71). En cuanto a la ocupación de la víctima decimos que aquellas que son trabajadores independientes tienen menos chance de sufrir violencia sexual que las víctimas que tienen otros tipos de ocupaciones (IC: $0,03-0,78$ ).

El coeficiente de determinación indica que el $38 \%$ de la variación en la violencia sexual esta explicada por los factores incluidos en el modelo.

La prueba de bondad de ajuste del modelo $\left(X^{2}=45 ; p=0,000\right)$, indica que por lo menos uno de los factores antes mencionados explica significativamente la violencia sexual.

La evaluación de la capacidad de pronóstico del modelo ajustado, indica que el $96 \%$ de las víctimas de violencia sexual fueron clasificados correctamente como víctimas de violencia sexual, mientras que el $41 \%$ de las personas que no han sufrido violencia sexual fueron clasificados correctamente por el modelo. En resumen el modelo clasificó correctamente el $88 \%$ de las víctimas de violencia sexual.

\section{Discusión}

Actualmente uno de los fenómenos a nivel nacional que constituye un grave problema para la salud pública, la economía y el desarrollo de los pueblos, que se instala de manera silenciosa en numerosas familias y deja sus terribles secuelas es la violencia conyugal, que en este estudio abarca la violencia física, psicológica y sexual.

Según Barja [1] que realizó una investigación con los datos de la ENDES en el 2015 encontró que el $66 \%$ de las mujeres sufrían de violencia psicológica. Otro estudio con los datos de la ENDES 2013 de Gonzales [4] , señala la prevalencia de violencia global en 44,5\%, la frecuencia de violencia psicológica, física y sexual fue de $30,3 \%, 36,8 \%$, y 8,6\%, respectivamente. Mientras que Blitchtein y Reyes [3] en su estudio hallaron una prevalencia de violencia física a nivel nacional de 14,5\%. Sin embargo, en la presente investigación existieron tendencias similares con todos los tipos de violencia, pero diferencias con otras investigaciones, esto puede ser que se deba a que nuestra base de datos proviene del Centro emergencia mujer que es una institución donde acuden las personas a denunciar que han sufrido algún tipo de violencia física, psicológica o sexual o puede ser que hayan sufrido dos tipo de violencia o las tres juntas.

Martínez [5] en su estudio obtuvo como resultados que las mujeres de baja escolaridad y amas de casa fueron las principales víctimas de violencia psicológica. Por otro lado, Blitchtein y Reyes [3], demostró que la condición de conviviente y tener mayor nivel educacional que la pareja son factores asociados a la violencia física contra la mujer. La presente investigación encontró que el factor psicológico presenta asociación con la violencia conyugal y los tres tipos de violencia por separado ya sea en alguna de sus categorías: trastornos de personalidad, entorno de alcoholismo y drogadicción y autoestima inadecuada que en este estudio fue la categoría de referencia; además el factor sociológico en su categoría influencia de medios masivos en referencia a la categoría incomunicación conyugal presentó asociación con la violencia conyugal. Estas diferencias en los resultados se pueden presentar debido a los distintos contextos sociales que vive que cada 
población estudiada, quedando en evidencia que la violencia es un problema multicausal que se expresa en todas las dimensiones sociales sin discriminar alguna.

Noblega y Muñoz [6] en su estudio cualitativo menciona que desde la perspectiva de las participantes los comportamientos machistas generaban conductas violentas de la pareja sobre ellas. Lo cual demuestra que en nuestra sociedad la equidad de género sigue siendo una brecha muy amplia en la que trabajar. Noblega y Virginia mostraron en su estudio cualitativo al alcohol como un factor que puede ocasionar graves discusiones con sus parejas, llegando a tener conductas violentas, además que influye indirectamente en la aparición de la violencia, debido al gasto en la economía del hogar que tiene. Así mismo Nóblega y Muñoz [6] encontró que cuando el hombre consume alcohol se torna agresivo. A pesar de algunas asociaciones, el rol exacto que cumple el consumo de alcohol en la violencia hacia la pareja se encuentra aún en debate y es posible que actúe como un desencadenante por lo que existirían otros factores asociados. Para la presente investigación el factor Consumo de alcohol que está dentro del factor psicológico se presentó significativamente asociado a la violencia conyugal y a los tres tipos de violencia física, sexual y psicológica, por lo tanto vemos que es un fuerte predictor de violencia. Una de las posibles explicaciones es que si bien a nivel social se ha concientizado sobre el consumo de alcohol a la población, se ha enfatizado más en otros sectores como en el de transporte, más no en el sector salud y su impacto en la familia y comunidad. Si bien el factor sociológico con su categoría familia patriarcal, no es significativo con la violencia conyugal si lo es con la violencia física y psicológica, esto es explicado en un estudio sobre la tolerancia social e institucional a la Violencia Basada en Género en Colombia en el cual se habla del Ordenamiento patriarcal en el que a la mujer se le asigna, por su cuestión de género, la protección y crianza de los hijos.

\section{Conclusiones}

- El factor psicológico, influye sobre la violencia conyugal y sobre los tres tipos de violencia: física, psicológica y sexual, vemos que es un factor influyente a nivel de la violencia en general.

- Los factores que resultaron significativos con la violencia conyugal son el factor psicológico y la ocupación de las víctimas. En el cuadro 3, se demuestra que las personas con trastornos de personalidad tienen 3 veces más posibilidad de sufrir violencia conyugal respecto a las personas a las personas que presentan autoestima inadecuada. Asimismo las personas en cuyo entorno hay alcohol y drogas tienen 24 veces más posibilidad de sufrir violencia conyugal que las personas tiene autoestima inadecuada.

En cuanto a la ocupación de la víctima, las personas que trabajan independientemente tienen menos chance de sufrir violencia conyugal respecto a las personas que tiene otros tipos de ocupación.

- El análisis multivariado de los factores en relación (ver cuadro 7) con la violencia física muestra que las personas en cuyo entorno hay alcoholismo y drogadicción tienen 6 veces más chance de sufrir violencia física que las personas que presentan autoestima inadecuada. Las personas que provienen de familias patriarcales tienen 25 veces más posibilidad de sufrir violencia física respecto a las personas con incomunicación conyugal.

- El análisis multivariado de los factores en relación con la violencia sexual muestra que las personas con trastornos de personalidad tiene 5 veces más chance de sufrir violencia sexual que las personas que sufren de autoestima inadecuada. Asimismo las personas en cuyo entorno hay alcohol y drogas tienen 10 veces más posibilidad de sufrir violencia sexual que las personas con autoestima inadecuada. 
Las personas que tienen influencia de medias masivas tienen menor posibilidad de sufrir violencia sexual respecto a las personas con incomunicación conyugal. Las personas que trabajan independientemente tienen menos posibilidad de sufrir violencia sexual respecto a las personas que tienen otros tipos de ocupaciones.

- El análisis multivariado de los factores en relación con la violencia psicológica muestra que las personas con trastornos de personalidad tienen 16 veces más posibilidad de sufrir violencia psicológica que las personas que tienen autoestima inadecuada. Además las personas en cuyo entorno hay alcohol y drogas tienen 25 veces más posibilidad de sufrir violencia psicológico que las personas que presentan autoestima inadecuada. Cuando hay presencia del factor cultural las mujeres que son discriminadas por su género tienen menor posibilidad de sufrir violencia psicológica respecto a las mujeres que son vistas como objeto sexual.

\section{Referencias bibliográficas}

[1] Barja, J. (2015). Factores que influyen en la violencia basada en género en mujeres de 20 a 40 años atendidas en el consultorio externo de ginecología del Hospital Sergio BernalesComas, noviembre diciembre 2015 (tesis de pregrado). Universidad Nacional Mayor de San Marcos, Lima, Perú.

[2] Arce, C. (2019). Factores asociados a la violencia conyugal en el centro de emergencia mujer (tesis de maestría). Universidad Nacional Mayor de San Marcos, Lima, Perú.

[3] Blitchtein, D. y Reyes, E. (2012). Factores asociados a la violencia física reciente de pareja hacia la mujer en el Perú. Revista Peruana de Medicina Experimental y Salud Pública, 29(1), $35-43$.

[4] Gonzales, C. (2013). Asociación entre violencia contra la mujer en relación de pareja y el nivel de riqueza. Perú 2013 (tesis de pregrado). Universidad Nacional Mayor de San Marcos, Lima, Perú.

[5] Martínez, R. (2015). La violencia doméstica en la mujer de la edad mediana. Hospital Docente Ginecobstétrico de Guanabac de La Habana, Cuba.

[6] Nóblega, M. y Muñoz, P. (2009). Una aproximación cualitativa a la violencia hacia la mujer en un asentamiento humano de Villa El Salvador. Liberabit 15(2), 95-107.

[7] Ochoa, C. (2015). Muestreo probabilístico: muestreo sistemático. Marketing and Innovation. Netquest. Recuperado de https://www.netquest.com/blog/es/blog/es/muestreo-porconveniencia

[8] Ramos, M. (2006). Masculinidades y violencia conyugal: Experiencias de vida de hombres de sectores populares de Lima y Cusco. Lima, Perú: Universidad Peruana Cayetano Heredia.

[9] Saldarriaga, C., Blass, M., y Hidalgo, T. (2013). Guía de atención integral de los centros "Emergencia Mujer". Lima, Perú: Ministerio de la Mujer y Poblaciones Vulnerables.

[10] Sanchéz, H., Blass, M., y Reyes, C. (2010). Metodologías y diseños en la investigación científica. Lima, Perú: Universidad Ricardo Palma. 